\title{
Frequency of Comorbid Musculoskeletal Problems in Patients with Parkinson's Disease: What and When?
}

\author{
Erdem Degirmenci $^{*}$ (1), Yildiz Degirmenci2 ${ }^{2}$ (i) \\ ${ }^{1}$ Duzce University Faculty of Medicine, Orthopedics and Traumatology Department, Duzce, Turkey \\ ${ }^{2}$ Duzce University Faculty of Medicine, Neurology Department, Duzce, Turkey \\ Email: *erddegir@gmail.com, ydegir@gmail.com
}

How to cite this paper: Degirmenci, E. and Degirmenci, Y. (2019) Frequency of Comorbid Musculoskeletal Problems in Patients with Parkinson's Disease: What and When? Neuroscience \& Medicine, 10, 331-338. https://doi.org/10.4236/nm.2019.104024

Received: September 10, 2019

Accepted: October 9, 2019

Published: October 12, 2019

Copyright $\odot 2019$ by author(s) and Scientific Research Publishing Inc. This work is licensed under the Creative Commons Attribution International License (CC BY 4.0).

http://creativecommons.org/licenses/by/4.0/

\begin{abstract}
Background: Musculoskeletal problems, deteriorating posture and pain are common complaints/comorbidities in Parkinson's disease (PD). The aim of this study was to investigate the frequency of comorbid musculoskeletal problems in $\mathrm{PD}$, and their relationship with disease stage and severity. Methods: The study was approved by the local ethical committee of Duzce University numbered with 2018/52 on date 26.03.2018. Patients with PD (pwPD) underwent a detailed examination to investigate the possible comorbidity of musculoskeletal findings in the orthopedics and traumatology clinic. Socio-demographic features, disease characteristics, motor and non-motor scores were recorded. Results: Thirty-seven pwPD were enrolled in the study. Mean age was $68.75 \pm$ 10.75 years. Comorbid musculoskeletal problems were rotator cuff syndrome (27\%), knee osteoarthritis (24.3\%), meniscus (13.5\%), lumber disc herniation (10.8\%), kyphosis (8.1\%), myotendinous diseases (16.3\%). Rotator-cuff disease and knee osteoarthritis were most common comorbidities, especially in the "mild" and "moderate" stages of pwPD. Conclusion: Since most of the pwPD, especially the ones that have musculoskeletal complaints prior to the diagnosis of $\mathrm{PD}$, present to the orthopedics and traumatology clinics or physiotherapists, it is mandatory to enhance the awareness of the orthopedic surgeons, and physiotherapy specialist in order to keep PD in mind.
\end{abstract}

\section{Keywords}

Parkinson's Disease, Musculoskeletal Disorders, Comorbidity, Rotator-Cuff Syndrome, Osteoarthritis

\section{Introduction}

Parkinson's disease (PD) is a chronic neurodegenerative disease which is prone 
to occur in the elderly, in the majority. Since it is known as the second most frequent neurodegenerative disease following Alzheimer's disease, PD is estimated to affect millions of people worldwide [1]. It has complex pathophysiology mainly affecting the basal ganglia. However, the hallmark pathology is considered to be the progressive loss of dopaminergic neurons in the substantia nigra pars compacta, in particular with the presence of intracytoplasmic eosinophilic inclusion bodies (Lewy bodies) in the remaining neurons in this region [1].

This progressive, disabling disease is characterized by cardinal characteristic features including bradykinesia, rigidity, rest tremor and postural instability. However, non-motor symptoms such as sleep disorders, cognitive, neuropsychiatric, autonomic, as well as sensory symptoms such as anosmia, fatigue, numbness, and pain can also deteriorate the daily lives of patients with PD (pwPD) [1] [2].

Not only pain, but also postural, bone-joint deformities have been accepted as the important aspects of the disease since the initial definition of the disease as "propensity to bend the trunk forward" by James Parkinson in "An Essay on the Shaking Palsy", 1817 [3] [4]. So far, review of the literature revealed the comorbidity of musculoskeletal problem (MSP) such as kyphosis, scoliosis which may even be considered as the motor manifestation of the disease itself, and camptocormia, Pisa syndrome, osteoporotic bone fractures, frozen shoulder, joint pain as well [5] [6] [7] [8] [9].

Regarding the comorbidity of various MSPs leading to disabling conditions in pwPD, the aim of our study was to investigate the comorbid MSPs in pwPD, and to evaluate their relationship with the disease stage and severity.

\section{Materials and Methods}

Patients with new and/or prior diagnoses of idiopathic Parkinson's Disease (IPD) who were recruited from the Neurology Department of our university hospital, and referred to the Orthopedics and Traumatology outpatient clinic for a detailed examination to investigate the possible comorbidity of musculoskeletal findings and diseases. The exclusion criteria were the diseases other than idiopathic PD such as Parkinson-plus syndromes (Multiple system atrophy, progressive supranuclear palsy, corticobasal degeneration, lewy body dementia), secondary parkinsonism including vascular parkinsonism due to stroke, druginduced parkinsonism, toxic-metabolic parkinsonism, patients who were unable to provide information, dementia and history of antipsychotic use. All patients were informed about the content of the study and gave their written approval before enrolment. The study was approved by the local ethical committee of Duzce University numbered with 2018/52 on date 26.03.2018.

Socio-demographic features of the pwPD were recorded, as well as the disease characteristics including the duration of the disease, and the initial symptom at onset. To assess the stage and severity of PD, Hoehn and Yahr ( $\mathrm{H} \& \mathrm{Y})$ stages with the Unified Parkinson's Disease Rating Scale (UPDRS) motor and non-motor scores of pwPD were recorded [10] [11] [12]. 
Musculoskeletal evaluations of the pwPD were performed by the same clinician with face-to-face interviews and findings and symptoms were recorded.

Data were organized in an SPSS Version 22 (Statistical Package for Social Sciences) database. Statistical analyses were performed with Kruskal-Walis $\mathrm{H}$ test for numerical variables and, Fisher-Freeman-Halton test for categorical variables to compare the groups. $\mathrm{P}$ values below 0.05 were considered to indicate statistical significance.

\section{Results}

Thirty-seven pwPD (22 women, 15 men) were enrolled the study. Socio-demographic features of the pwPD are shown in Table 1.

The initial symptom types of pwPD are shown in Figure 1.

Mean duration of the onset of the symptoms of disease was $74.70 \pm 49.86$ months (range $=1-192$ months), corresponding to 6 years approximately. All pwPD were under combined dopaminergic treatments, and under follow-up in the work beginning from the year 2018.

Mean $\mathrm{H} \& \mathrm{Y}$ score of the pwPD was $2.00 \pm 1.02$ (ranges = 1 - 5). In assessing the disease severity, $\mathrm{H} \& \mathrm{Y}$ scores 1 to 2 are considered as "mild" stages of the disease, while 3 as "moderate", and 4 to 5 are accepted as "severe" disease [13]. According to the H \& Y stages, most of our pwPD ( $\mathrm{n}=30$, 81.1\%) had "mild" PD. However, 4 (10.8\%) had "moderate", and 3 (8.1\%) had "severe" PD.

Mean UPDRS motor score of pwPD was $32.59 \pm 14.46$ (ranges $=12-60$ ), and non-motor score was $8.32 \pm 5.09$ (ranges $=0-19$ ). Since we assessed the motor and non-motor symptom severity of the pwPD, the cut-off score for the mild/moderate non-motor UPDRS score was 10/11, while it was $21 / 22$ for the moderate/severe PD. These cut-off scores were 32/33 for mild/moderate motor UPDRS scores, and 58/59 for moderate/severe motor UPDRS scores [13].

Medical history assessment of the patients in terms of orthopedic problems,

Table 1. Socio-demographic features of the pwPD.

\begin{tabular}{cccccc}
\hline \multirow{2}{*}{ Mean age (years old) } & Mean BMI* & \multicolumn{4}{c}{ Occupation (n, \%) } \\
\cline { 3 - 5 } & & Retired & Housewife & Officer & Self-employed \\
\hline $\begin{array}{c}68.75 \pm 10.75 \text { years } \\
\text { (ranges }=45-88 \text { years) }\end{array}$ & $102.66 \pm 444.11$ & $20,54.1 \%$ & $14,(37.8 \%)$ & $1(2.7 \%)$ & $2(5.4 \%)$ \\
\hline
\end{tabular}

${ }^{*}$ BMI: Body mass index.

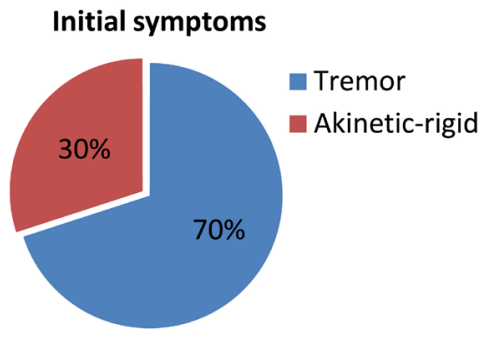

Figure 1. Initial symptom type of pwPD. 
$13(35.1 \%)$ had no previous musculoskeletal and/or orthopedic complaints. However, $8(21.6 \%)$ had previous lumbar disc herniation, 8 (14.3\%), 5 (13.5\%) had knee problems, 3 (8.1\%) had shoulder, 2 (5.4\%) had cervical, 2 (5.4\%) had back and knee, $1(2.7 \%)$ had hip, $1(2.7 \%)$ had shoulder and knee problems. The remaining 2 (5.4\%) had other problems causing nonspecific pain.

Even if the majority as $35.1 \%$ reported no history of any orthopaedic problems, orthopedic and musculoskeletal evaluations of all pwPD revealed that all had some kind of an orthopedic and/or MSP leading to a clinical diagnosis. The most contributing factor to musculoskeletal problems in PD was considered as the duration of disease. These were rotator cuff $(n=10,27 \%)$, knee osteoarthritis $(n=$ $9,24.3 \%)$, meniscus $(\mathrm{n}=5,13.5 \%)$, lumber disc herniation $(\mathrm{n}=4,10.8 \%)$, kyphosis $(n=3,8.1 \%)$, and myotendinous diseases (MTDs) $(n=6,16.3 \%)$.

The presence of MSPs and diagnosis in association with the onset of disease, as well as the disease severity and stage are summarized in Table 2.

There were no statistically significant differences between the disease duration and the existence of MSPs ( $\mathrm{p}>0.05$ ).

As can be seen in Table 1, all orthopedics and MSPs were prone to occur in the mild stages of $\mathrm{PD}$, that corresponds to stage 1 and 2 in $\mathrm{H} \& \mathrm{Y}$ staging, rather than the late and severe stages of the disease $(\mathrm{p}>0.05)$ (Table 1$)$.

Rotator-cuff disease and knee osteoarthritis were most common orthopaedic diagnosis, especially in the "mild" and "moderate" stages of pwPD, with the disease duration of 5.5 and 7 years, respectively ( $p>0.05)$.

Kyphosis was the earliest MSP that mostly occurred in pwPD with the median disease duration of 3 years. However, knee osteoarthritis, meniscus, and MTDs were found to be emerged as the disease duration prolonged to 6 to 7 years ( $p>$ 0.05). Moreover, in the patients with "severe" PD that corresponds to stages 4 and 5 in $\mathrm{H} \& \mathrm{Y}$ staging, the most common MSPs were rotator-cuff disease, and MTDs ( $>$ > 0.05) (Table 1$)$.

Table 2. Musculoskeletal diagnosis in association with the onset of disease, as well as the disease severity and stage.

\begin{tabular}{|c|c|c|c|c|c|c|c|}
\hline & $\begin{array}{l}\text { Rotator cuff } \\
\quad(n=10)\end{array}$ & $\begin{array}{c}\text { Knee } \\
\text { osteoarthritis } \\
(n=9)\end{array}$ & $\begin{array}{l}\text { Meniscus } \\
(n=5)\end{array}$ & $\begin{array}{l}\mathrm{LDH}^{\star} \\
(\mathrm{n}=4)\end{array}$ & $\begin{array}{l}\text { Kyphosis } \\
(\mathrm{n}=3)\end{array}$ & $\begin{array}{l}\text { MTDs }^{\star *} \\
(n=6)\end{array}$ & $\mathrm{p}$ \\
\hline Median disease onset; months (range) & $66(8-180)$ & $84(24-192)$ & $72(24-96)$ & $59.5(1-120)$ & $36(18-96)$ & $72(24-168)$ & 0.805 \\
\hline Median UPDRS ${ }^{* * *}$ motor score (range) & $30(14-59)$ & $44(12-55)$ & $20(17-31)$ & $41(12-44)$ & $28(15-32)$ & $38(17-60)$ & 0.245 \\
\hline Median UPDRS ${ }^{* * *}$ non-motor score (range) & $8.5(1-17)$ & $8(3-19)$ & $7(0-8)$ & $10(2-15)$ & $2(2-17)$ & $6(2-16)$ & 0.568 \\
\hline \multicolumn{7}{|l|}{$\mathrm{HY}^{* * * *}$ score; $\mathrm{n}(\%)$} & \multirow{6}{*}{0.705} \\
\hline 1 & $4(40.0)$ & $2(22.2)$ & $4(80.0)$ & $1(25.0)$ & $1(33.3)$ & $0(0.0)$ & \\
\hline 2 & $4(40.0)$ & $5(55.6)$ & $1(20.0)$ & $2(50.0)$ & $2(66.7)$ & $4(66.7)$ & \\
\hline 3 & $0(0.0)$ & $2(22.2)$ & $0(0.0)$ & $1(25.0)$ & $0(0.0)$ & $1(16.7)$ & \\
\hline 4 & $1(10.0)$ & $0(0.0)$ & $0(0.0)$ & $0(0.0)$ & $0(0.0)$ & $0(0.0)$ & \\
\hline 5 & $1(10.0)$ & $0(0.0)$ & $0(0.0)$ & $0(0.0)$ & $0(0.0)$ & $1(16.7)$ & \\
\hline
\end{tabular}

${ }^{\star}$ Lumbar disc herniation; ${ }^{* *}$ Myotendinous diseases; ${ }^{* *}$ Unified Parkinson’s Disease Rating Scale; ${ }^{\star * * *}$ Hoehn and Yahr. 
When we evaluated association of the MSPs with the median UPDRS non-motor symptom scores of the pwPD, there were no statistically significant differences between the musculoskeletal diagnosis, and the median scores were found to be similar, varying from 6 to 8.5 ( $\mathrm{p}>0.05$ ). However, median UPDRS-non motor score was as lower as 2 , in patients with the diagnosis of kyphosis, which was also consistent with a shorter duration of PD ( $p>0.05)$.

\section{Discussion}

PD is the second most common neurodegenerative disease, following Alzheimer's disease in the elderly population, particularly. This chronic, progressive disease mainly affecting extrapyramidal system causes motor cardinal symptoms as rest tremor, rigidity, bradykinesia and postural instability manifests itself with a classical stooped posture, in which increased kyphosis, flexed hips and knees may occur [1] [2] [14].

Regarding the pathophysiology of PD, musculoskeletal symptoms and findings deteriorating posture, and spine such as frozen shoulder, osteoporosis, osteoporotic bone fractures, kyphosis, scoliosis, Pisa syndrome, camptocormia and pain are common complaints and comorbidities in pwPD [15] [16]. They may either occur in the prodromal phase of PD before the motor symptoms emerge, that encounters the very early stages of the disease, or in the later stages as the disease progress. Moreover, when the symptoms like pain, and frozen shoulder and many others occur before the motor symptoms of PD in the prodromal phase of the disease, most of the patients present to the orthopedic or physiotherapy clinics to seek help, and treatment unaware of PD [16] [17].

Based on these aspects, our primary outcome was to evaluate the frequency of comorbid musculoskeletal findings in pwPD, and to define their diagnosis in terms of musculoskeletal and/or orthopedic diseases. Our secondary outcome was to determine whether they occur in the mild or severe stages of the disease.

As we know from the literature, rotator cuff syndrome is one of the commonest findings in pwPD either in the early or late stages of the disease [15]. In a previous study by Koh et al., rotator cuff disease was reported in the $70 \%$ of the pwPD (22 out of 33), and these patients had a longer duration of PD [18]. Twenty five (10 out of 37 ) of our pwPD had rotator cuff syndrome, and the median disease duration was of our pwPD experiencing rotator cuff syndrome was 5.5 years, which was relatively a short duration, and the severity of PD was consistent with mild/moderate PD with a median UPDRS motor score of 30 . Similar to the literature revealing rotator-cuff syndrome as a prodromal or early finding of PD, in mild stages, most of our pwPD suffering from rotator-cuff syndrome also had mild PD, in terms of $\mathrm{H} \& \mathrm{Y}$ stages of 1 and 2. Though, we found rotator-cuff syndrome as an early comorbid MSP in PD [13].

Osteoarthritis of the knee joint was the second most common comorbid orthopedic problem in our pwPD with a rate of $26.8 \%$. The patients experiencing 
osteoarthritis of the knee joint had the longest duration of $\mathrm{PD}$ as 7 years, had the highest median UPDRS motor scores as 44 in consistent with moderate/severe $\mathrm{PD}$, and were in the mild to moderate stage according to $\mathrm{H} \& \mathrm{Y}$. As we know from the literature, osteoarthritis, traumatic arthritis, and other types of joint disorders can also be seen in PD [16]. There is limited data about the frequency of osteoarthritis in PD from Rana et al. [19], and in this study. They reported 34 patients with osteoarthritis out of 68 patients with $\mathrm{PD}$. Mean duration of PD was approximately 4 years which was shorter than the disease duration of our pwPD, and the patients had mild to moderate PD according to the UPDRS motor scores, and $\mathrm{H} \& \mathrm{Y}$ scores as well [19].

As another postural deformity in pwPD related to the classical stooped posture, kyphosis in patients with camptocormia is a disabling musculoskeletal comorbidity in pwPD, most prone to occur as the disease progresses with long disease durations, and moderate to severe PD [20] [21]. Differing from the literature, only $8.1 \%$ of our pwPD had the diagnosis of kyphosis and these were the ones with the shorter disease duration, and mild/moderate disease. This inconsistency with the literature may be due to our small number of participants, which is the limitation of our study.

As the consequences of our study revealed the comorbidity of musculoskeletal problems deteriorating the quality of life in pwPD, clinicians including orthopaedic and traumatology, neurologists, physiotherapists, as well as the general practitioners should work in a multidisciplinary practice to contribute the early diagnosis of PD which should lead to the reduction of the influence of musculoskeletal problems via introducing the precise treatments.

\section{Conclusion}

Musculoskeletal problems and complaints are an important reason for disability and/or deterioration in many pwPD. Since most of the pwPD, especially the ones that have musculoskeletal complaints prior to the diagnosis of $\mathrm{PD}$, present to the orthopedics and traumatology outpatient clinics as well as the physiotherapist, it is mandatory to enhance the awareness, and knowledge of the orthopedic surgeons, and physiotherapy specialist in order to keep PD in mind. However, further research with large number of participants that address these musculoskeletal conditions in pwPD should be beneficial to establish precisely, and multidisciplinary approach, and treatment options before leading to disability, which are still limited.

\section{Acknowledgements}

The authors were solely responsible for the conception and performance of this study and the writing of this manuscript.

\section{Authors' Contributions}

ED was responsible for the conception and design of the study. ED was respon- 
sible for acquisition of data. YD performed the data analysis. ED and YD drafted the manuscript. All authors participated in interpretation of the findings and approved the final version of the manuscript. All authors confirm that the content has not been published elsewhere and does not overlap with or duplicate their published work.

\section{Conflicts of Interest}

The authors declare no conflicts of interest regarding the publication of this paper.

\section{References}

[1] Zesiewicz, T.A. (2019) Parkinson Disease. Continuum, 25, 896-918. https://doi.org/10.1212/CON.0000000000000764

[2] Chaudhuri, K.R., Healy, D.G. and Schapira, A.H. (2006) Non-Motor Symptoms of Parkinson's Disease: Diagnosis and Management. National Institute for Clinical Excellence. The Lancet Neurology, 5, 235-245.

https://doi.org/10.1016/S1474-4422(06)70373-8

[3] Chaudhuri, K.R. and Jenner, P. (2017) Two Hundred Years since James Parkinson's Essay on the Shaking Palsy-Have We Made Progress? Insights from the James Parkinson's 200 Years Course Held in London, March 2017. Movement Disorders, 32, 1311-1315. https://doi.org/10.1002/mds.27104

[4] Donaldson, I.M. (2015) James Parkinson's Essay on the Shaking Palsy. The Journal of the Royal College of Physicians of Edinburgh, 45, 84-86. https://doi.org/10.4997/JRCPE.2015.118

[5] Baik, J.S., Kim, J.Y., Park, J.H., et al. (2009) Scoliosis in Patients with Parkinson's Disease. Journal of Clinical Neurology, 5, 91-94.

https://doi.org/10.3988/jen.2009.5.2.91

[6] Djaldetti, R., Mosberg-Galili, R., Sroka, H., et al. (1999) Camptocormia (Bent Spine) in Patients with Parkinson's Disease Characterization and Possible Pathogenesis of an Unusual Phenomenon. Movement Disorders, 14, 443-447. https://doi.org/10.1002/1531-8257(199905)14:3<443::AID-MDS1009>3.0.CO;2-G

[7] Tassorelli, C., Furnari, A., Buscone, S., et al. (2012) Pisa Syndrome in Parkinson's Disease: Clinical, Electromyographic, and Radiological Characterization. Movement Disorders, 27, 227-235. https://doi.org/10.1002/mds.23930

[8] Schneider, J.L., Fink, H.A., Ewing, S.K., et al. (2008) Study of Osteoporotic Fractures (SOF) Research Group. The Association of Parkinson's Disease with Bone Mineral Density and Fracture in Older Women. Osteoporosis International, 19, 1093-1097. https://doi.org/10.1007/s00198-008-0583-5

[9] Riley, D., Lang, A.E., Blair, R.D., et al. (1989) Frozen Shoulder and Other Shoulder Disturbances in Parkinson's Disease. Journal of Neurology, Neurosurgery, and Psychiatry, 52, 63-66. https://doi.org/10.1136/jnnp.52.1.63

[10] Hoehn, M.M. and Yahr, M.D. (1967) Parkinsonism: Onset, Progression and Mortality. Neurology, 17, 427-442. https://doi.org/10.1212/WNL.17.5.427

[11] Goetz, C.G., Poewe, W., Rascol, O., et al. (2004) Movement Disorder Society Task Force Report on the Hoehn and Yahr Staging Scale: Status and Recommendations. Movement Disorders, 19, 1020-1028. https://doi.org/10.1002/mds.20213

[12] Movement Disorder Society Task Force on Rating Scales for Parkinson's Disease 
(2003) The Unified Parkinson's Disease Rating Scale (UPDRS): Status and Recommendations. Movement Disorders, 18, 738-750. https://doi.org/10.1002/mds.10473

[13] Martínez-Martín, P., Rodríguez-Blazquez, C., Alvarez, M., et al. (2015) Parkinson’s Disease Severity Levels and MDS-Unified Parkinson's Disease Rating Scale. Parkinsonism and Related Disorders, 21, 50-54. https://doi.org/10.1016/j.parkreldis.2014.10.026

[14] Ashour, R. and Jankovic, J. (2006) Joint and Skeletal Deformities in Parkinson's Disease, Multiple System Atrophy, and Progressive Supranuclear Palsy. Movement Disorders, 21, 1856-1863. https://doi.org/10.1002/mds.21058

[15] Papalia, R., Torre, G., Papalia, G., et al. (2019) Frozen Shoulder or Shoulder Stiffness from Parkinson Disease? Musculoskeletal Surgery, 103, 115-119. https://doi.org/10.1007/s12306-018-0567-3

[16] Rabin, M.L., Earnhardt, M.C., Patel, A., et al. (2016) Postural, Bone, and Joint Disorders in Parkinson's Disease. Movement Disorders Clinical Practice, 18, 538-547. https://doi.org/10.1002/mdc3.12386

[17] Frandsen, R., Kjellberg, J., Ibsen, R., et al. (2014) Morbidity in Early Parkinson's Disease and Prior to Diagnosis. Brain and Behavior, 4, 446-452.

https://doi.org/10.1002/brb3.228

[18] Koh, S.B., Roh, J.H., Kim, J.H., et al. (2008) Ultrasonographic Findings of Shoulder Disorders in Patients with Parkinson's Disease. Movement Disorders, 23, 1772-1776. https://doi.org/10.1002/mds.22191

[19] Rana, A.Q., Qureshi, A.R., Akhter, S., et al. (2018) Osteoarthritis Increases Paresthestic and Akathisic Pain, Anxiety Case-Ness, and Depression Severity in Patients with Parkinson's Disease. Frontiers in Neurology, 13, 409. https://doi.org/10.3389/fneur.2018.00409

[20] Charpentier, P., Dauphin, A., Stojkovic, T., et al. (2005) Parkinson's Disease, Progressive Lumbar Kyphosis and Focal Paraspinal Myositis. Revista de Neurología (Paris), 161, 459-463. https://doi.org/10.1016/S0035-3787(05)85077-5

[21] Wadia, P.M., Tan, G., Munhoz, R.P., et al. (2011) Surgical Correction of Kyphosis in Patients with Camptocormia Due to Parkinson's Disease: A Retrospective Evaluation. Journal of Neurology, Neurosurgery, and Psychiatry, 82, 364-368.

https://doi.org/10.1136/jnnp.2009.176198 